\title{
A Multi-Material Virtual Prototyping System for Biomedical Applications
}

\author{
S.H. Choi ${ }^{1}$, H.H. Cheung ${ }^{2}$, W.K. Zhu ${ }^{3}$ \\ Department of Industrial and Manufacturing Systems Engineering \\ The University of Hong Kong, Hong Kong \\ 1shchoi@hkucc.hku.hk \\ 2hhcheung@graduate.hku.hk; ${ }^{3}$ h0795413@hkusua.hku.hk
}

\begin{abstract}
This paper describes a multi-material virtual prototyping (MMVP) system for modelling and digital fabrication of discrete and functionally graded multi-material objects for biomedical applications. The MMVP system consists of a DMMVP module, an FGMVP module, and a virtual reality (VR) simulation module. The DMMVP module is used for design and process planning of discrete multi-material (DMM) objects, while the FGMVP module is for functionally graded multimaterial (FGM) objects. The VR simulation module integrates these two modules to perform digital fabrication of multimaterial objects, which can be subsequently visualized and analyzed in a virtual environment to optimize MMLM processes for fabrication of product prototypes. Using the MMVP system, two biomedical objects, including a human dextrocardic heart made of discrete multi-materials and a hip joint assembly of FGM are modelled and digitally fabricated for visualization and analysis in a VR environment. These studies show the MMVP system is a practical tool for modelling, visualization, process planning, and subsequent fabrication of biomedical objects of discrete and functionally graded multi-materials for biomedical applications.
\end{abstract}

Keywords - Biomedical objects; multi-material layered manufacturing; virtual prototyping;

\section{INTRODUCTION}

It has been commonly recognized that Layered Manufacturing (LM) is a potential technology for fabrication of biomedical objects, such as artificial hip joints, organs, tissue scaffolds, and bone and jaw structures, for studying and planning complex surgical operations, implantations, and as specimens for experiments in pharmaceutical manufacturing enterprises or hospitals. Wang et al. [1] developed a precision extruding deposition (PED) system to fabricate interconnected 3D scaffolds at Drexel University.

However, most commercial LM systems can only fabricate single material objects which cannot meet the needs for biomedical applications. A typical example of dental implantation requires a dental implant to be made of functionally graded multi-material (FGM) structures composed of titanium (Ti) and hydroxyapatite (HAP), in order to satisfy both mechanical and biocompatible property requirements [2]. Therefore, it is desirable and necessary to develop Multi-Material Layered Manufacturing (MMLM) technology for fabrication of multi-material biomedical objects.
Multi-material (or heterogeneous) objects may be classified into two major types, namely (i) discrete multi-material (DMM) objects with a collection of distinct materials, and (ii) functionally graded multi-material (FGM) objects with materials that change gradually from one type to another. In comparison with single-material objects, a DMM object can clearly differentiate one part from others, or tissues from blood vessels of a human organ, while an FGM object can perform better in rigorous environments. In particular, suitably graded composition transitions across multi-material interfaces can create an object of very different properties to suit various applications $[3,4]$.

Some researchers have explored using LM to fabricate multi-material prototypes. A few experimental multi-material layered manufacturing (MMLM) machines, such as a discrete multiple material selective laser sintering ( $\left.\mathrm{M}^{2} \mathrm{SLS}\right)$ machine $[5,6]$, a shape deposition manufacturing machine [7,8], a fused deposition of multiple ceramics (FDMC) machine [9], and a 3D inkjet-printing machine [10-12] have been developed. Although these systems seemed suitable for relatively simple objects of a limited variety of materials, they provided a good foundation for further hardware development. The development of MMLM is perhaps more of a software issue than of a hardware problem; it is essential to develop an integrated software system for modelling and fabrication of complex multi-material objects.

In order to fabricate multi-material prototypes, both material and geometric information must be made available. Although STL is now a de-facto industrial standard file format for LM, it only contains geometric information. Therefore, some researchers have proposed CAD representation methods for DMM objects or FGM objects to facilitate general CADCAM applications, including MRPII [13-20]. Some of these representation methods, when fully developed and widely adopted, would be useful for MMLM. However, most proposed methods need relatively large memory, and cannot conveniently represent complex multi-material objects, particularly for biomedical applications.

Indeed, a significant proportion of complex objects, particularly human organs and bone structures etc, are not designed using CAD systems. Instead, they are captured by laser digitizers, or CT, MRI, and micro CT scanners. Sun et al. [21] reviewed the use of $\mathrm{CT}$ and MRI techniques to model 
tissue scaffolds as CAD models that can be used for biomimetic design, analysis, simulation, and freeform fabrication of the tissue scaffolds. In general, the digitized images are normally processed to form a model in STL format with no material or topological information needed to extract the slice contours. Indeed, slice contours are random in nature without any explicit topological hierarchy relationship, and to process them for multi-toolpath planning remains a challenging obstacle that has yet to be surmounted. Most of the above representation schemes cannot conveniently represent objects from CT and MRI images, and subsequent processing of multi-material objects for fabrication control is ignored. Hence, it is worthwhile to develop an integrated computer system to represent and process multi-material biomedical objects for subsequent generation of toolpaths for fabrication control.

This paper therefore describes a multi-material virtual prototyping (MMVP) system for modelling, visualization, and digital fabrication of discrete and functionally graded (FG) multi-material objects for biomedical applications. The MMVP system offers flexibility in representing such objects from either CAD models or CT/MRI scan images. It also provides a virtual reality (VR) environment for digital fabrication, visualization, and quality analysis of multimaterial biomedical prototypes. As such, the need for physical prototyping is minimized, and the cost and time of biomedical product development reduced substantially. Hence, the MMVP system provides a practical tool for engineers, doctors, and surgeons to design and visualize biomedical objects for study and planning of surgical operations or dental implantations; it can also be adapted for control of MMLM machines for physical fabrication of multi-material objects in manufacturing and medical applications.

\section{THE MMVP SYSTEM}

\section{A. The DMMVP Module}

The DMMVP is one of the main modules of the MMVP system, consisting of a suite of software packages for design and visualization of multi-material objects and simulation of MMLM process. These packages includes a colour modeller for colouring monochrome STL models, a slicing algorithm, a topological hierarchy-sorting algorithm for grouping random slice contours of DMM objects, a topological hierarchy-based toolpath planning algorithm for generation of sequential and concurrent multi-toolpaths, and a virtual prototyping (VP) module for digital fabrication of DMM objects. It works as follows.

Firstly, a biomedical model created by CAD or an MRI/CT digitizer is converted into STL format, which is the industry de-facto standard. As STL is monochrome or single-material, an in-house package is used to paint the STL model, with each colour representing a specific material.
Secondly, a few steps are undertaken to prepare for subsequent simulation of the MMLM process and visualization of the resulting digital prototypes: (a) slice the colour STL model into a number of layers of a predefined thickness. The resulting layer contours and material information are stored in a modified Common Layer Interface (CLI) file; (b) sort the slice contours with a contour sorting algorithm to establish explicit topological hierarchy; (c) based on the hierarchy information, multi-toolpath planning algorithms are used to plan and generate multi-toolpaths by hatching the slice contours with a predefined hatch space. The hatch vectors are stored in the modified CLI file for fabrication of digital prototypes and build-time estimation.

Thirdly, the VP module performs digital fabrication of multi-material prototypes. The resulting digital prototypes can be stereoscopically visualized and analyzed to review and improve the design of the biomedical objects efficiently.

The following section uses a human dextrocardic heart to demonstrate how the DMMVP module can model and fabricate multi-material objects for biomedical applications, such as surgical training and planning, patient's education, and implantations. Dextrocardia is a heart disease in which the primitive heart tube folds to the left in a mirror image of a normal bulboventricular loop.
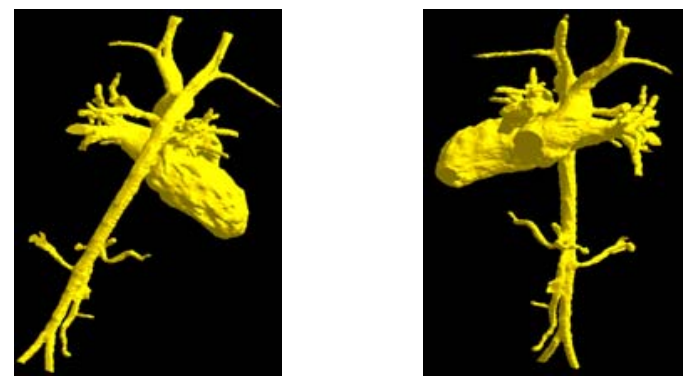

Figure 1. A monochrome human dextrocardic heart STL model

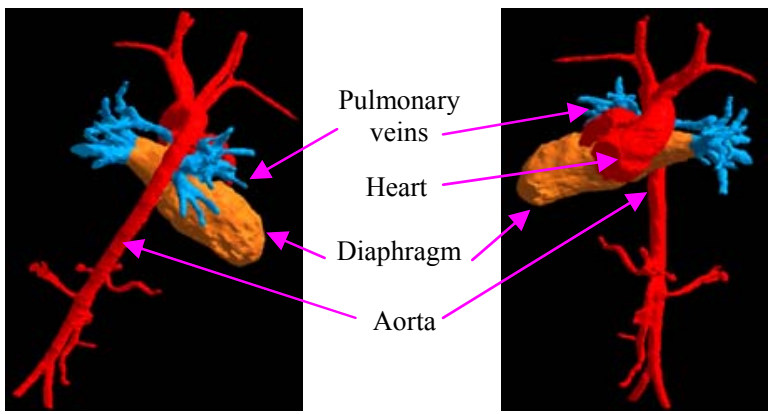

Figure 2. A colour human dextrocardic heart STL model for users to vividly visualize various parts of the heart

Fig. 1 shows a monochrome human dextrocardic heart STL model constructed from CT or MRI images. Obviously, using the monochrome STL model, it may not be easy for users to differentiate various parts of the heart. To solve this, the colour STL modeller is used to paint the human dextrocardic heart STL model as a colour STL model, as 
shown in Fig. 2. In comparison with the monochrome STL model, surgeons can visualize and differentiate the various parts of the dextrocardic heart more vividly to explain and plan complex surgical operations. Moreover, each colour represents a specific type of material, and hence a colour STL model can provide both geometric and material information for process planning of MMLM process.

To fabricate this heart prototype with discrete multimaterials, a set of nozzles $\left(\mathrm{N}_{\mathrm{i}}, \mathrm{i}=1,2, \ldots \mathrm{n}\right)$ would deposit specific materials on appropriate slice contours. It is necessary to identify and relate specific contours of a slice to a particular tool and subsequently arrange the toolpaths to fabricate the prototype efficiently. This requires a multi-toolpath planning algorithm to generate toolpaths without tool collisions. However, most multi-material objects tend to be complex and the slice contours do not possess any explicit topological hierarchy relationship. As a result, it is very difficult to associate specific contours with a particular tool. To tackle this problem, a topological hierarchy-based approach to toolpath planning for MMLM was proposed by the authors $[22,23]$. This approach adopts a topological hierarchy-sorting algorithm to construct the topological hierarchy in terms of a parent-and-child list that defines the containment relationship of the contours of a slice. Thus, with the hierarchy relationship, it is no longer necessary to identify and relate contours to a particular nozzle one by one for multi-toolpath planning. Indeed, only grouping of the outermost contours is required. Besides, parametric polygons are used to construct tool envelopes for contour families with the same material property to simplify detection of tool collisions during concurrent movements of nozzles. Hence, concurrent toolpaths without redundant movements and collisions can be easily generated for controlling MMLM machines to fabricate physical multi-material prototypes.

A layer containing 12 contours, as shown in Fig. 3, is to be made of three kinds of materials, namely $\mathrm{m}_{1}, \mathrm{~m}_{2}$, and $\mathrm{m}_{3}$. Three nozzles, $\mathrm{N}_{1}, \mathrm{~N}_{2}$, and $\mathrm{N}_{3}$, are allocated to deposit specific materials, $\mathrm{m}_{1}, \mathrm{~m}_{2}$, and $\mathrm{m}_{3}$, respectively. Fig. 4 shows the topological hierarchy relationship of the contours in Fig. 3. According to the contour families, ten toolpaths namely, $\mathrm{P}_{\mathrm{C} 1}$, $\mathrm{P}_{\mathrm{C} 2}, \mathrm{P}_{\mathrm{C} 3}, \mathrm{P}_{\mathrm{C} 4}, \mathrm{P}_{\mathrm{C} 5,10,11}, \mathrm{P}_{\mathrm{C} 6}, \mathrm{P}_{\mathrm{C} 7}, \mathrm{P}_{\mathrm{C} 8}, \mathrm{P}_{\mathrm{C} 9}$, and $\mathrm{P}_{\mathrm{C} 12}$ are generated. Subsequently, the toolpaths with the same material properties are grouped together to form three toolpath-sets, namely $S_{1}$ to $S_{3}$, which are associated with three nozzles from $\mathrm{N}_{1}$ to $\mathrm{N}_{3}$, respectively. Three work envelopes from $\mathrm{E}_{1}$ to $\mathrm{E}_{3}$ for each of these nozzles are constructed to facilitate planning of concurrent multi-toothpaths. Hence, using the hierarchy information and association relationship between the toolpathsets and the nozzles, concurrent toolpaths without redundant tool movements and collisions can be easily generated and planned for fabrication control.

With the toolpaths, the heart prototype can now be digitally fabricated using the VP module [24, 25]. Fig. 5 shows the digital fabrication process of a few layers of the heart.

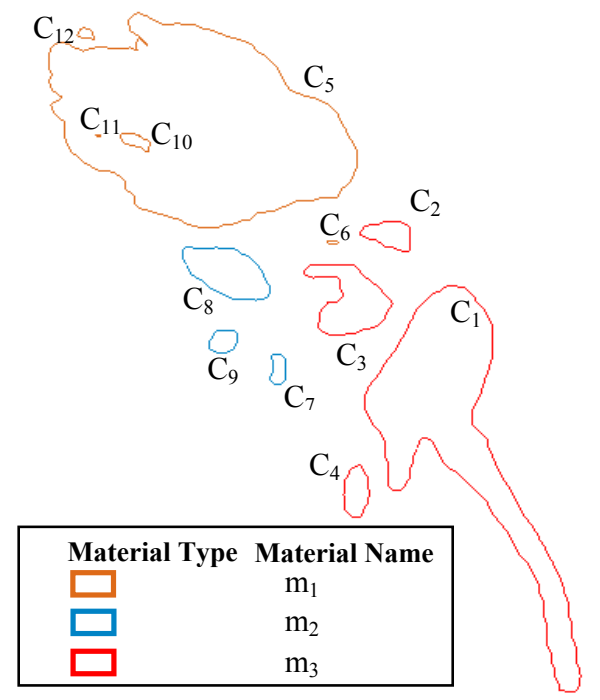

Figure 3. A slice of a colour STL model - a human heart with dextrocardia

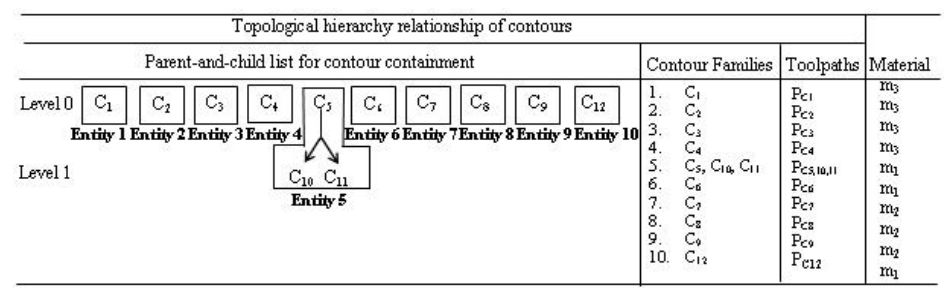

Figure 4. Topological hierarchy relationship of the heart model

After fabrication, the resulting DMM heart prototype can be manipulated in a full-immersive VR environment, as shown in Fig. 6, to visualize the quality of the prototype that the MMLM machine will subsequently deliver. A build-time estimation algorithm has been developed to estimate the fabrication time [26]. Besides, dimensional deviations of the prototype beyond a tolerance limit can be identified by superimposing the colour heart STL model on its digital prototype. As such, biomedical engineers can conveniently perform design iterations to improve the surface texture of the resulting prototype. Thus, an optimal combination of process parameters, such as layer thickness, build direction, and hatch space can be obtained for cost effective fabrication of physical biomedical prototypes.

\section{B. The FGMVP Module}

To repair or replace failing organs or tissues due to trauma or aging, biomedical prototypes may have to be made of functionally graded materials to mimic biological and mechanical characteristics of the organs or tissues. To achieve this, the system is enhanced to represent and fabricate FGM objects. The following section describes the FGMVP module for modelling and fabrication of FGM objects in detail. 
The FGMVP module is used for modelling and fabrication of FGM objects. It is characterized by a contour-based FGM modeller, in which an FGM object is represented by material control functions and discretization of layer contours with topological hierarchy. Material control functions are specified across contour families of some representative layers in X-Y plane and across layers along Z-axis. The material composition at any location is calculated from control functions, and the slice contours are discretized into subregions of constant material composition. The discretization resolution can be varied to suit display and fabrication requirements.

Firstly, the FGMVP module slices a monochrome STL model obtained from a traditional CAD design or digitized images, and sorts the resulting contours to build explicit topological hierarchy information.

Secondly, the contours are loaded into the FGMVP module for FGM object representation, with the following steps: (1) select a number of feature contour families in a representative layer; (2) specify control functions for material variations across layers along Z-axis in the build direction; (3) specify control functions for material variations in X-Y plane; and (4) discretize the slice contours into sub-regions of constant material composition.

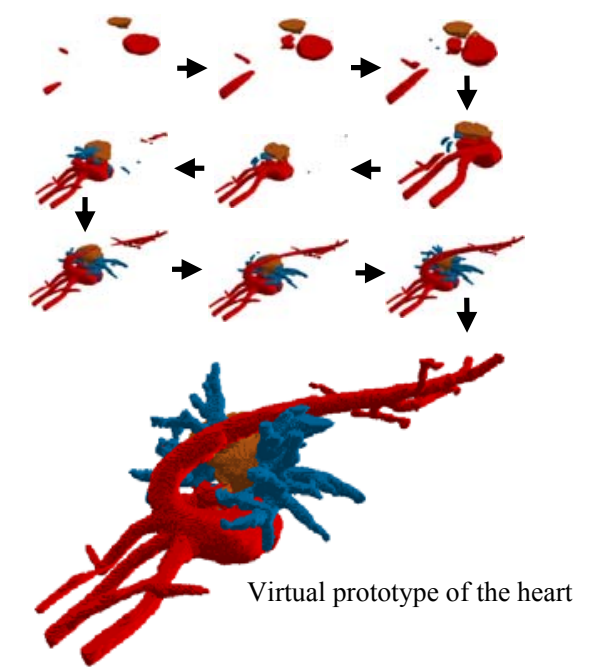

Figure 5. Digital fabrication process of a DMM human heart

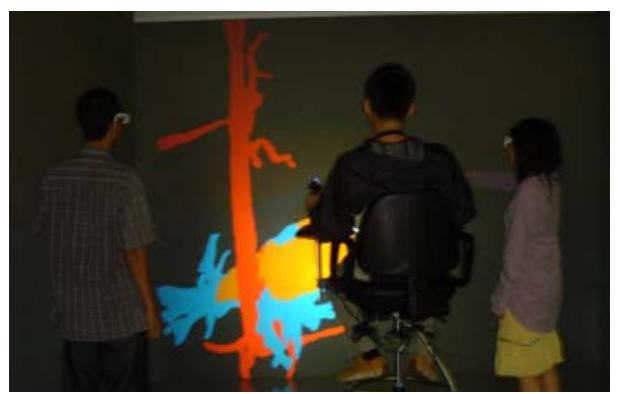

Figure 6. Immsersive VR environment for stereoscopic visualization and analysis of the DMM heart prototype
Thirdly, the resulting contour-based FGM model containing both geometric and material composition variation information is processed for visualization, analysis, and fabrication of FGM objects.

In comparison with voxel-based representation schemes, this approach is computationally efficient and requires little memory for processing relatively complex objects. More importantly, it facilitates physical fabrication on MMLM machines. The detail of the contour-based FGM modeller was presented in $[27,28]$. In the following sections, a hip joint is processed to illustrate the use of the FGMVP module as a practical tool for design and fabrication of FGM biomedical objects.

Fig. 7 shows an artificial joint assembly for hip prosthesis [29]. To fabricate such a joint, the FGM components have to be represented accordingly. Fig. 8 shows a monochrome STL hip-joint assembly model with two components, namely a stem and a femoral ball head. For better medical properties, the stem should have a biocompatible material at the outer surface and a mechanically tough material at the core. Similarly, the femoral ball head should have a wear-resistant outer surface and a mechanically tough core.

Using the FGMVP module, the STL hip-joint assembly model is firstly sliced into a set of layers as a homogeneous contour-based model as in Fig. 9, and the topological hierarchy information of each layer is established; secondly, a feature layer is selected for assigning primary materials and material control functions for calculation of property values of material composition; thirdly, each layer is discretized into sub-regions of constant material composition. Subsequently, the resulting geometric contours and material information are used for visualization and process planning of MMLM process.

Fig. 10 shows the resulting FGM layers in a wireframe and a rendered display mode, respectively. For the stem, it has a yellow/black graded variation in $\mathrm{X}-\mathrm{Y}$ to represent a gradual change of material composition from hydroxyapatite at the surface to titanium at the core, giving the desirable biocompatible properties at the surface and the desirable mechanical properties at the core. For the femoral ball head, it has a blue/black graded variation in X-Y plane to represent a gradual change of material composition from an alumina at the surface to a zirconia at the core, providing a wear-resistant outer surface and a tough core.

Moreover, the discretization resolution can be easily changed accordingly to control the smoothness of material composition variations. Fig. 11 shows a finer material composition variation compared with the one in Fig. 10. The digital fabrication process of the FGM hip-joint assembly prototype is shown in Fig. 12. This shows that the FGMVP module is a practical tool for design of FGM objects and simulation of MMLM process for biomedical applications. 


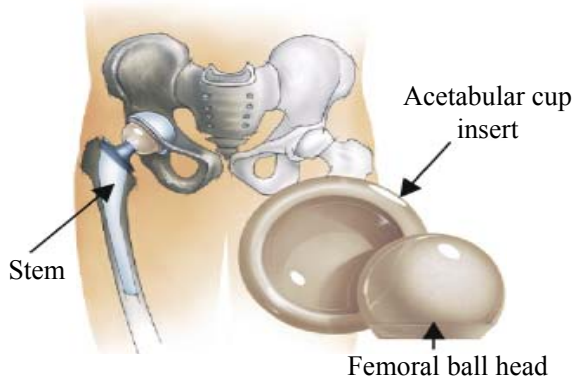

Figure 7. An artificial joint for hip prosthesis [29]

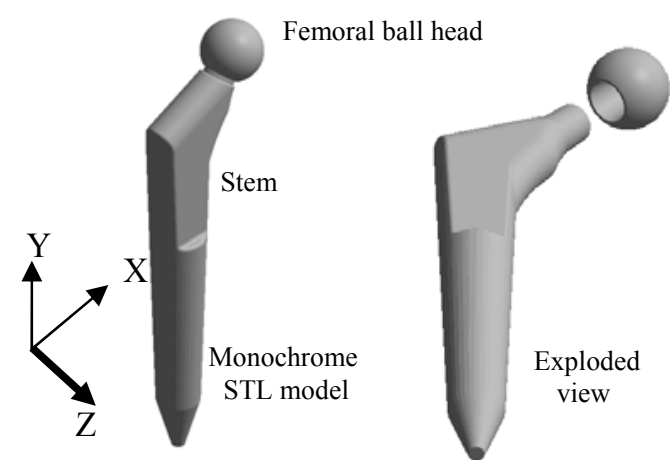

Figure 8. An assembly with a femoral ball head and a stem for hip replacement prothesis

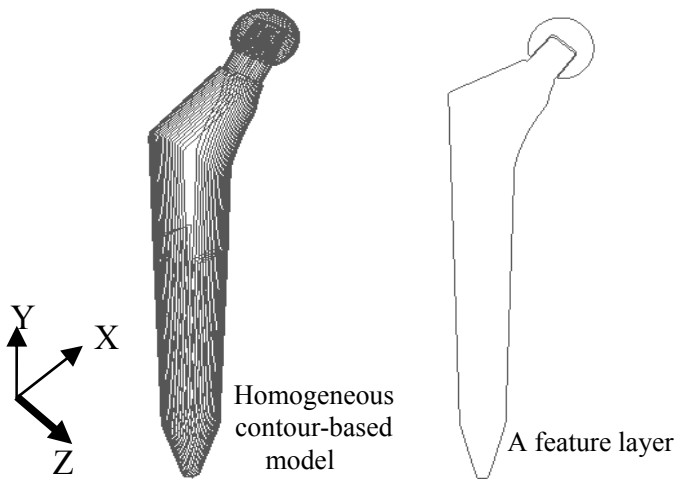

Figure 9. A homogeneous contour-based hip-joint assembly and a feature layer for assigning primary materials and material control functions

\section{The VR Simulation Module}

The DMMVP module and the FGMVP module above are integrated with a virtual reality (VR) simulation module to form the MMVP system for modelling and digital fabrication of discrete and functionally graded multi-material objects for biomedical applications. The VR simulation module can be executed in a semi- or a full-immersive platform to take advantage of affordability or high level of immersiveness to facilitate product design and digital fabrication of multimaterial objects [22, 24, 25]. Through simulations, design validation and modification of a biomedical product can be iterated without incurring any manufacturing and material costs of physical prototyping. Therefore, the cost and time of product development can be reduced considerably.

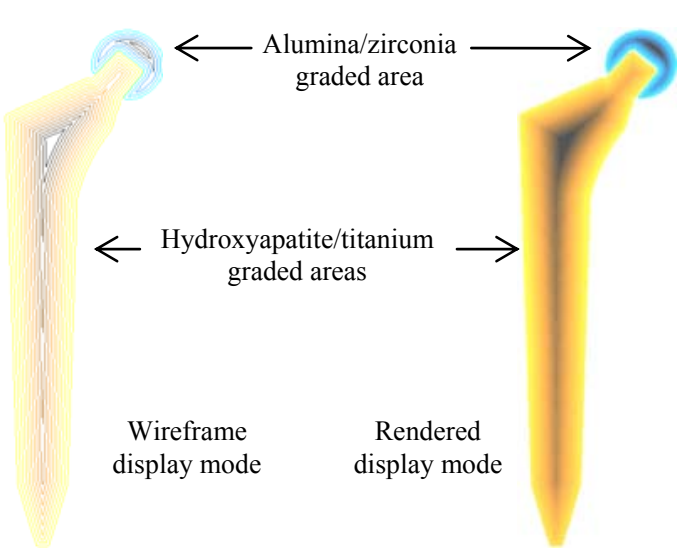

Figure 10. The resulting FGM layer of the hip-joint assembly in Fig. 9

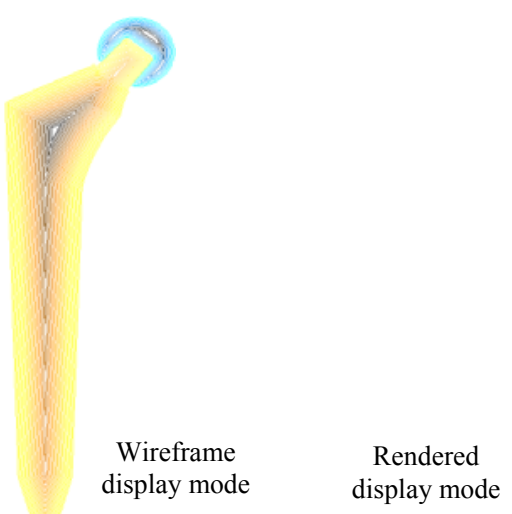

Figure 11. The layer with a finer material composition variation compared with the FGM layer

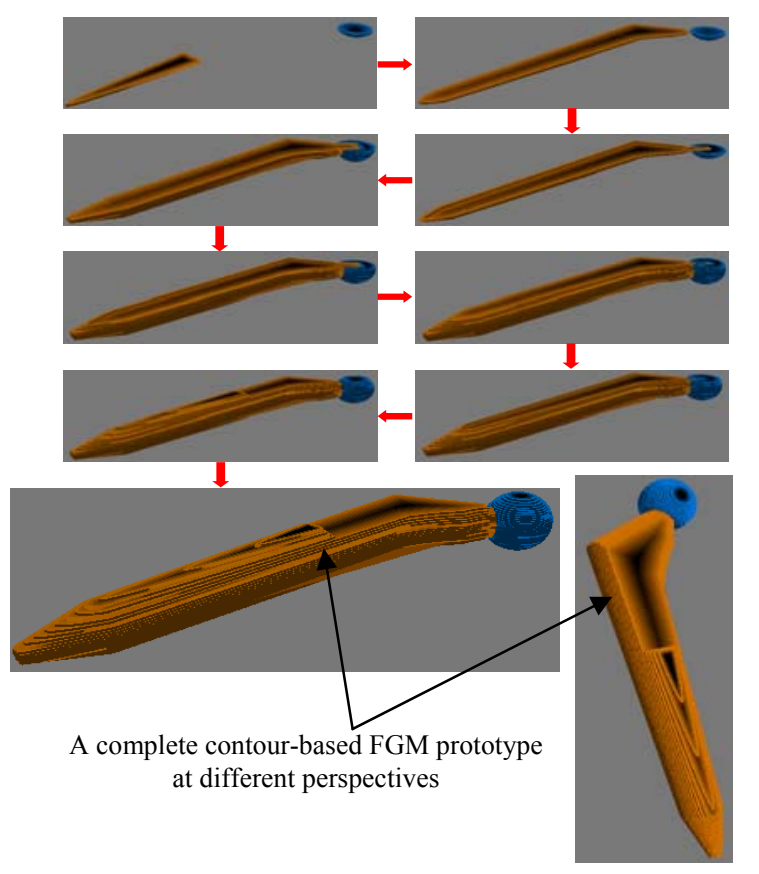

Figure 12. Digital fabrication of an FGM hip-joint assembly prototype 


\section{CONCLUSION}

This paper presents a multi-material virtual prototyping (MMVP) system for modelling, process planning, and digital fabrication of discrete and functionally graded multi-material objects for biomedical applications. The system is characterized by its topological hierarchy-based toolpath planning algorithm and contour-based approach to representation of FGM objects. Case studies showed that the system can conveniently process CAD models and CT/MRI images to represent complex multi-material objects for biomedical and dental applications. It is computationally efficient and requires relatively little memory for processing complex objects. More importantly, it facilitates visualization of the resulting multi-material objects in a VR environment and their subsequent physical fabrication on MMLM machines.

\section{ACKNOWLEDGMENT}

The authors would like to acknowledge the Research Grant Council of the Hong Kong SAR Government and the CRCG of the University of Hong Kong for their financial support for this project.

\section{REFERENCES}

[1] G. F. Wang, L. Shor, A. Darling, S. Khalil, W. Sun, S. Güçeri, A. Lau, Precision extruding deposition and characterization of cellular poly- $\varepsilon$ caprolactone tissue scaffolds, Rapid Prototyping Journal 10(1) (2004) 420-429.

[2] F, Watari, A. Yokoyama, F. Saso, M. Uo, T. Kawasaki, Fabrication and properties of functionally graded dental implant, Composites Part B 28B (1997) 5-11.

[3] V. Kumar, Solid modeling and algorithms for heterogeneous objects, Ph.D. Thesis, The University of Michigan, USA, (1999).

[4] K.H. Shin, D. Dutta, Constructive representation of heterogeneous objects, Journal of Computing and Information Science in Engineering 1(3) (2001) 205-217.

[5] J. Jepson, J.J. Beaman, D.L. Bourell, K.L. Wood, SLS processing of functionally gradient materials, in: D.L. Bourell, et al. (Eds.), Solid Freeform Fabrication Symposium, Austin, Texas. The University of Texas, 1(997), pp. 1-8.

[6] K. Lappo, B. Jackson, K. Wood, D.L. Bourell, J.J. Beaman, Discrete multiple material selective laser sintering $\left(\mathrm{M}^{2} \mathrm{SLS}\right)$ : experimental study of part processing, in: D.L. Bourell, et al. (Eds.), Solid Freeform Fabrication Symposium, Austin, Texas, The University of Texas, (2003), pp. 109-119.

[7] R. Merz, F.B. Prinz, K. Ramaswami, M. Terk, L.E. Weiss, Shape deposition manufacturing, in: D.L. Bourell, et al. (Eds.), Solid Freeform Fabrication Symposium, Austin, Texas, The University of Texas, (1994), pp. 1-8.

[8] J.R. Fessler, A.H. Nickel, G.R. Link, F.B. Prinz, P. Fussell, Functional gradient metallic prototypes through shape deposition manufacturing, in: D.L. Bourell, et al. (Eds.), Solid Freeform Fabrication Symposium, Austin, Texas, The University of Texas, (1997), pp. 521-528.

[9] M.A. Jafari, W. Han, F. Mohammadi, A. Safari, S.C. Danforth, N. Langrana, A novel system for fused deposition of advanced multiple ceramics, Rapid Prototyping Journal 6(3) (2000) 161-175.

[10] T.R. Jackson, H. Liu, N.M. Patrikalakis, E.M. Sachs, M.J. Cima, Modeling and designing functionally graded material components for fabrication with local composition control, Materials \& Design 20(2-3) (1999) 63-75.

[11] W.J. Cho, E.M. Sachs, N.M. Patrikalakis, D.E. Troxel, A dithering algorithm for local composition control with three-dimensional printing, Computer-Aided Design 35(9) (2003) 851-867.

[12] J.W. Wang, L.L. Shaw, Fabrication of functionally graded materials via inkjet color printing, Journal of the American Ceramic Society 89(10) (2006) 3285-3289.

[13] V. Kumar, S. Rajagopalan, M. Cutkosky, D. Dutta, Representation and processing heterogeneous objects for solid freeform fabrication. The $6^{\text {th }}$ IFIP WG 5.2 International Workshop on Geometric Modeling: Fundamentals and Applications, Tokyo, Japan. The University of Tokyo, 1998.

[14] S.M. Morvan, G.M. Fadel, Heterogeneous solids: possible representation schemes. In: Bourell D.L., et al., editors. Solid Freeform Fabrication Symposium, Austin, Texas, The University of Texa, (1999), pp. 187198.

[15] W.K. Chiu, S.T. Tan, Multiple material objects: from CAD representation to data format for rapid prototyping, Computer-Aided Design 32(12) (2000) 707-717.

[16] C.T. Hsieh, N.A. Langrana, A system approach in extrusion-based multi-material CAD, in: Bourell, D.L., el al., editors, Solid Freeform Fabrication Symposium, Austin, Texas, The University of Texas, (2001), pp, 313-321.

[17] T.R. Jackson, Analysis of functionally graded material object representation methods, Ph.D. Thesis, Massachusetts Institute of Technology, USA, (2000).

[18] K.H. Shin, Representation and process planning for layered manufacturing of heterogeneous objects, Ph.D. Thesis, The University of Michigan, USA (2002).

[19] X.Y. Kou, S.T. Tan, Heterogeneous object modeling: a review, Computer-Aided Design 39(4) (2007) 284-301.

[20] K. Samanta, B. Koc, Feature-based design and material blending for free-form heterogeneous object modeling, Computer-Aided Design 37(3) (2005) 287-305.

[21] W. Sun, B. Starly, J. Nam, A. Darling, Bio-CAD modeling and its applications in Computer-aided tissue engineering, Computer-Aided Design 37(11) (2005) 1097-111.

[22] S.H. Choi, H.H. Cheung, A multi-material virtual prototyping system, Computer-Aided Design 37(1) (2005) 123-136.

[23] S.H. Choi, H.H. Cheung, A topological hierarchy-based approach to toolpath planning for multi-material layered manufacturing, ComputerAided Design 38(2) (2006) 143-156.

[24] S.H. Choi, H.H. Cheung, A CAVE-based multi-material virtual prototyping system, Computer-Aided Design and Applications 3(5) (2006) 557-566.

[25] S.H. Choi, H.H. Cheung, A versatile virtual prototyping system for rapid product development, Computers in Industry 59(5) (2008) 477-488.

[26] S.H. Choi, H.H. Cheung, Multi-material virtual prototyping for product development and biomedical engineering, Computers in Industry 58(5) (2007) 438-452.

[27] H.H. Cheung, A versatile multi-material virtual prototyping system, Ph.D. Thesis, The University of Hong Kong, Hong Kong, (2007).

[28] S.H. Choi, H.H. Cheung, A topological hierarchy-based approach to layered manufacturing of functionally graded multi-material objects, Computers in Industry (2009), doi:10.1016/j.compind.2009.01.008.

[29] G. Anné, K. Vanmeensel, J. Vleugels, O.V.D. Biest, Electrophoretic deposition as a novel near net shaping technique for functionally graded biomaterials, in: O.V. D. Biest, et al. editors, The $8^{\text {th }}$ International Symposium on Multifunctional and Functionally graded Materials (FGM 2004), Leuven, Belgium, July 11-14, (2005) pp. 213-218. 